\title{
Reconstruction of cracks and material losses by perimeter-like penalizations and phase-field methods: numerical results
}

\author{
WOLFGANG RING \\ Institute of Mathematics, University of Graz, Heinrichstr. 36, 8010 Graz, Austria \\ E-mail: wolfgang.ring@uni-graz.at \\ LUCA RONDI \\ Dipartimento di Matematica e Informatica, Università degli Studi di Trieste, \\ via Valerio 12/1, 34127 Trieste, Italy \\ E-mail:rondi@units.it
}

[Received 9 June 2010 and in revised form 19 April 2011]

\begin{abstract}
We numerically implement the variational approach for reconstruction in the inverse crack and cavity problems developed by one of the authors. The method is based on a suitably adapted freediscontinuity problem. Its main features are the use of phase-field functions to describe the defects to be reconstructed and the use of perimeter-like penalizations to regularize the ill-posed problem.

The numerical implementation is based on the solution of the corresponding optimality system by a gradient method. Numerical simulations are presented to show the validity of the method.
\end{abstract}

2010 Mathematics Subject Classification: Primary 35R30; Secondary 65N21, 65K10.

Keywords: Inverse problems; cracks; cavities; phase-field; perimeter penalization; optimality system.

\section{Introduction and setting of the method}

We consider a homogeneous and isotropic conducting body, assumed to be contained in $\Omega$, a bounded, Lipschitz domain of $\mathbb{R}^{N}, N \geqslant 2$. We assume that there exist $\Omega_{1}$, a Lipschitz domain contained in, and different from, $\Omega$, and a closed set $\gamma \subset \partial \Omega \cap \partial \Omega_{1}$ such that the interior of $\gamma$ is not empty and $\gamma$ has a positive distance from $\Omega \backslash \overline{\Omega_{1}}$. We assume that $\gamma$ is known and accessible to measurements.

In the body some defects might be present, which we assume to be perfectly insulating and outside $\Omega_{1}$. Namely, we model these defects by a closed set $K_{0} \subset \bar{\Omega}$ such that $K_{0} \cap \overline{\Omega_{1}}$ is empty. A minimal assumption on $K_{0}$ is that the $(N-1)$-dimensional Hausdorff measure of $K_{0}$ is finite. We denote by $G_{K_{0}}$ the connected component of $\Omega \backslash K_{0}$ containing $\Omega_{1}$, that is, the region of $\Omega$ reachable from $\gamma$ without crossing $K_{0}$.

The defects may have different geometrical properties. For instance, we may have, even at the same time, cracks (either interior or surface-breaking), or material losses (either interior, that is, cavities, or at the boundary).

We say that a defect $K_{0}$ is a material loss if $G_{K_{0}}$ coincides with the interior of its closure. In this case no cracks are present and $K_{0}$ represents the boundary of the material loss. For instance, if the defect is given by a single cavity $\sigma_{0}=\overline{D_{0}}$, where $D_{0}$ is a domain compactly contained in $\Omega$ and such that $\Omega \backslash \sigma_{0}$ is connected, then $K_{0}=\partial \sigma_{0}$ and $G_{K_{0}}=\Omega \backslash \sigma_{0}$. The other connected component of $\Omega \backslash K_{0}$ in this case is simply given by $D_{0}$. In Figure 1 we illustrate the geometric configuration in the case when we have at the same time two cracks and one cavity. 


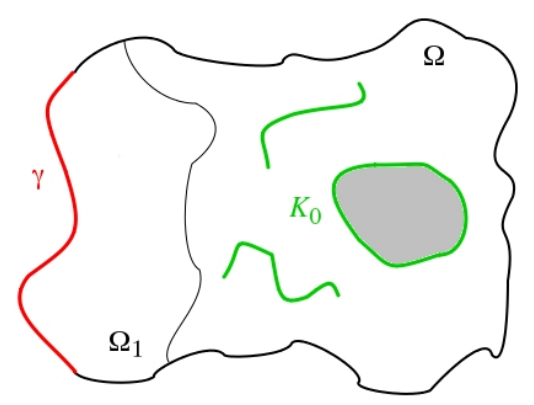

FIG. 1. Geometric configuration.

Let us consider the following experiment. If a current density $f_{0}$ is applied on $\gamma$, then the electrostatic potential in $\Omega, u_{0}=u\left(f_{0}, K_{0}\right)$, is the solution to the following (normalized) Neumann boundary value problem:

$$
\begin{cases}\Delta u=0 & \text { in } \Omega \backslash K_{0}, \\ \nabla u \cdot v=f_{0} & \text { on } \gamma, \\ \nabla u \cdot v=0 & \text { on } \partial\left(\Omega \backslash K_{0}\right) \backslash \gamma, \\ \int_{\gamma} u=0 . & \end{cases}
$$

The current density is modeled by a function $f_{0} \in L^{s}(\gamma)$, for some constant $s>N-1$, such that $\int_{\gamma} f_{0}=0$. The electrostatic potential $u_{0}$ may then be measured on $\gamma$. We call such a measurement $g_{0}=\left.u_{0}\right|_{\gamma}$ and we observe that $g_{0} \in L^{2}(\gamma)$ and $\int_{\gamma} g_{0}=0$. In this way we obtain an electrostatic boundary measurement of voltage, $g_{0}$, and current, $f_{0}$, type on $\gamma$. In mathematical words, we measure the Cauchy data $\left(g_{0}, f_{0}\right)$ of the harmonic function $u_{0}$ on $\gamma$. Clearly, for the prescribed current $f_{0}$, the voltage $g_{0}$ depends on $K_{0}$. If $K_{0}$ is unknown, then the measured voltage $g_{0}$ may provide information about the unknown defect. In fact, the aim of the inverse problem is to reconstruct an unknown defect $K_{0}$ by prescribing one or more current densities $f_{0}$ and measuring the corresponding value of the potentials on $\gamma$. Such a problem arises, for instance, in non-destructive evaluation, to determine flaws like cracks or cavities in conducting bodies by non-invasive methods. We refer to this problem as the inverse crack problem, in the general case. Instead, when we a priori know that the defect is a material loss, we call it the inverse cavity problem. For results on the inverse crack problem and related problems, we refer to the review article [7], where uniqueness, stability and reconstruction results are reviewed. We wish to mention that more recently new numerical methods have been devised for the reconstruction of cracks, although in the case of perfectly conducting ones [5, 4].

Here we simply wish to note that a single measurement (that is, performing the experiment previously described only once) is enough to determine uniquely a material loss, in any dimension. In the general crack case, instead, one measurement may not be enough, but two suitably chosen measurements (corresponding to two suitable prescribed current densities) are enough for unique identification of any kind of defect, at least in the planar case. The prescribed current densities that give uniqueness in the two-dimensional case are of the following type. We consider three electrode locations on the boundary. At one of these locations we keep fixed a positive electrode, while the 
negative one is placed alternatively at one of the other two locations. This kind of configuration is applied in numerical experiments, where four electrode locations are used, each on a different side of the computational domain. For each measurement, we place the positive electrode at one location and the negative one at another.

Let us finally remark that if the unknown defect is a priori assumed to be interior (that is, $K_{0} \subset \Omega$ ) and if the whole boundary of $\Omega$ is accessible and connected, then we may simply take $\gamma=\partial \Omega$. This is the assumption we make for numerical experiments.

Our approach to this inverse problem is the following. We observe that $u_{0}$ is smooth outside $K_{0}$, whereas it may, and generally does, jump across $K_{0}$. Therefore, starting from the Cauchy data, we wish to reconstruct the function $u_{0}$ in $\Omega$, and in particular its discontinuity set $J\left(u_{0}\right)$. We notice that this is not a classical Cauchy problem for $u_{0}$, since $u_{0}$ is harmonic in $\Omega \backslash K_{0}$ with $K_{0}$ unknown! Rather, it looks more like a free-discontinuity problem for $u_{0}$, since its discontinuity set $J\left(u_{0}\right)$ is unknown and it is actually the aim of our reconstruction. If we are able to reconstruct $u_{0}$ and $J\left(u_{0}\right)$, then we obtain valuable information on $K_{0}$, given that $J\left(u_{0}\right) \subset K_{0}$. Actually, for the inverse cavity problem, $J\left(u_{0}\right)$ determines the whole $\partial G_{K_{0}}$. On the contrary, in the inverse crack problem, it may happen that a crack is not visible for a particular measurement, that is, $J\left(u_{0}\right)$ does not detect the whole $\partial G_{K_{0}}$. In this case, we may change the prescribed current density, reconstruct again the electrostatic potential from its values on $\gamma$, and recover another portion of $\partial G_{K_{0}}$. The uniqueness results tell us how many times and with which kind of prescribed current densities we need to repeat this procedure to fully reconstruct the unknown defect.

The main difficulties in the reconstruction of $u_{0}$ from its Cauchy data are the following. First of all, the problem is severely ill-posed, as Cauchy problems for elliptic equations are. Second, since the potential $u_{0}$ to be reconstructed is a discontinuous function whose discontinuities are unknown (actually they are the aim of our reconstruction), the problem is not even linear. Thus all the main difficulties of the original inverse problem are still present in the reconstruction of $u_{0}$.

The way to tackle ill-posedness is crucial. In fact, since the boundary data are measured, the data which are really available are not the exact Cauchy data $\left(g_{0}, f_{0}\right)$ but some noisy perturbation of them.

As mentioned above, rather than a classical Cauchy problem, we consider such a reconstruction as a free-discontinuity problem for the unknown potential $u_{0}$. For further details on freediscontinuity problems and their approximations we refer for instance to $[1,6]$. We follow the variational approach developed in $[10,11]$ for cracks and material losses, respectively. Such a method is based on the following two features. The first one is the choice of regularization. In order to regularize the problem, a perimeter-like penalization is used. Namely, we penalize the $(N-1)$ dimensional measure of the unknown defect $K_{0}$ (actually of the discontinuity set of the unknown potential). Second, the discontinuity sets are modeled through phase-field functions.

In this paper we develop the numerics of the approach in $[10,11]$. Let us describe in detail the method we plan to use.

We recall that we have fixed $\Omega$ and $\Omega_{1}$, two bounded, Lipschitz domains of $\mathbb{R}^{N}, N \geqslant 2$, such that $\Omega_{1}$ is a proper subset of $\Omega$. We also fix a closed set $\gamma \subset \partial \Omega \cap \partial \Omega_{1}$ such that the interior of $\gamma$ is not empty and $\gamma$ has a positive distance from $\Omega \backslash \overline{\Omega_{1}}$.

Assumptions on the prescribed current density

We fix a constant $s>N-1$. The prescribed current density will be denoted by $f_{0} \in L^{s}(\gamma)$; it satisfies $\int_{\gamma} f_{0}=0$. 


\section{Assumptions on $K_{0}$}

We assume that $K_{0}$ is a closed subset of $\bar{\Omega}$ such that $K_{0} \cap \overline{\Omega_{1}}$ is empty. We assume that the $(N-1)$ dimensional Hausdorff measure of $K_{0}$ is finite and that there exist a constant $q>2$ and a constant $C$, independent of $f_{0}$, such that

$$
\left\|\nabla u_{0}\right\|_{L^{q}(\Omega)} \leqslant C\left\|f_{0}\right\|_{L^{s}(\gamma)},
$$

where $u_{0}=u\left(f_{0}, K_{0}\right)$ solves (1.1).

The constant $q>2$ will be kept fixed throughout the paper and depends on very mild regularity properties of the defect $K_{0}$ to be reconstructed. We refer to Proposition 5.2 in [11] for a description of sufficient conditions under which (1.2) holds. Associated to $q$, we shall also need a constant $q_{1}$ defined as follows:

$$
0<q_{1}=(q-2) /(2 q)<1 / 2 \text {. }
$$

\section{Assumptions on the boundary data}

The measured potential at the boundary will be $g_{0}=\left.u_{0}\right|_{\gamma}$, where $u_{0}$ solves (1.1). We notice that $g_{0} \in L^{2}(\gamma)$ and $\int_{\gamma} g_{0}=0$. The available noisy data will be $\left(g_{\varepsilon}, f_{\varepsilon}\right)$ where $\varepsilon, 0<\varepsilon \leqslant 1 / 2$, denotes the noise level. We assume that $f_{\varepsilon}$ belongs to $L^{s}(\partial \Omega)$ and satisfies $\operatorname{supp}\left(f_{\varepsilon}\right) \subset \gamma$ and $\int_{\partial \Omega} f_{\varepsilon}=0$, whereas $g_{\varepsilon}$ belongs to $L^{2}(\gamma)$ and satisfies $\int_{\gamma} g_{\varepsilon}=0$. We assume that

$$
\left\|f_{0}-f_{\varepsilon}\right\|_{L^{s}(\gamma)} \leqslant \varepsilon \quad \text { and } \quad\left\|g_{0}-g_{\varepsilon}\right\|_{L^{2}(\gamma)} \leqslant \varepsilon \text {. }
$$

We shall use the following auxiliary functions.

Let $\psi: \mathbb{R} \rightarrow \mathbb{R}$ be a continuous and non-decreasing function such that $\psi(0)=0, \psi(t)>0$ if $t>0$, and $\psi(1)=1$. We assume that $\psi \in C^{1, \alpha}(\mathbb{R})$ for some exponent $\alpha, 0<\alpha \leqslant 1$, and that the $C^{1, \alpha}$ norm of $\psi$ is bounded. Furthermore, we require that for any $t \leqslant 0$ we have $\psi(t)=\psi(0)=0$, while $\psi(t)=\psi(1)=1$ for any $t \geqslant 1$. In particular, $\psi^{\prime}(0)=\psi^{\prime}(1)=0$. For example, we may set

$$
\psi(t)=-2 t^{3}+3 t^{2} \quad \text { for any } t \in[0,1]
$$

with straightforward extension beyond $[0,1]$.

Finally, for any $0<\varepsilon \leqslant 1 / 2$, we define

$$
\psi_{\varepsilon}=\left(1-\varepsilon^{2}\right) \psi+\varepsilon^{2}
$$

We introduce a single-well potential $V$ centered at 1 , that is, a non-negative continuous function such that $V(t)=0$ if and only if $t=1$. We assume that $V \in C^{1, \alpha}(\mathbb{R})$ and that its $C^{1, \alpha}$ norm is bounded. We also require that for any $t \leqslant 0$ we have $V(t) \geqslant V(0)$. Obviously, $V(t) \geqslant V(1)$ for any $t \geqslant 1$. For example, we may choose

$$
V(t)=(t-1)^{2} / 4 \quad \text { for any } t \in[0,1],
$$

with straightforward extension beyond $[0,1]$.

We shall also need a double-well potential $W$ centered at 0 and 1 , that is, a non-negative continuous function such that $W(t)=0$ if and only if $t \in\{0,1\}$. We assume that also $W \in C^{1, \alpha}(\mathbb{R})$ and that its $C^{1, \alpha}$ norm is bounded. Obviously, $W(t) \geqslant W(0)$ for any $t \leqslant 0$, and $W(t) \geqslant W(1)$ for any $t \geqslant 1$. For example, we may choose

$$
W(t)=9 t^{2}(t-1)^{2} \quad \text { for any } t \in[0,1],
$$

with straightforward extension beyond $[0,1]$. 
We define the space $H(\Omega)=\left\{\tilde{v} \in H^{1,2}(\Omega): \tilde{v}=0\right.$ a.e. in $\left.\Omega_{1}\right\}$. To any $\tilde{v} \in H(\Omega)$ we associate the function $v=1-\tilde{v}$. We remark that $v \in H^{1,2}(\Omega)$ and $v=1$ almost everywhere in $\Omega_{1}$.

We finally fix positive tuning parameters $a, b$ and $c$, and a noise level $\varepsilon, 0<\varepsilon \leqslant 1 / 2$. All these constants and the notation will be kept fixed throughout the paper.

The method is the following. We begin from the crack case and we propose to minimize, with respect to the phase-field variable $\tilde{v} \in H(\Omega)$ with the constraint $0 \leqslant \tilde{v} \leqslant 1$, the functional $\mathcal{F}_{\varepsilon}$ : $H(\Omega) \rightarrow \mathbb{R}$ defined as follows. For any $\tilde{v} \in H(\Omega)$, recalling that $v=1-\tilde{v}$, we set

$$
\mathcal{F}_{\varepsilon}(\tilde{v})=\frac{a}{\varepsilon^{q_{1}}} \int_{\gamma}\left|\tilde{u}_{\varepsilon}-g_{\varepsilon}\right|^{2}+b \int_{\Omega} \psi_{\varepsilon}(v)\left|\nabla \tilde{u}_{\varepsilon}\right|^{2}+\frac{c^{2}}{\varepsilon} \int_{\Omega} V(v)+\varepsilon \int_{\Omega}|\nabla v|^{2} .
$$

Here $\tilde{u}_{\varepsilon}=\tilde{u}_{\varepsilon}(\tilde{v})$ solves

$$
\begin{cases}\operatorname{div}\left(\psi_{\varepsilon}(v) \nabla \tilde{u}_{\varepsilon}\right)=0 & \text { in } \Omega, \\ \psi_{\varepsilon}(v) \nabla \tilde{u}_{\varepsilon} \cdot v=f_{\varepsilon} & \text { on } \partial \Omega, \\ \int_{\gamma} \tilde{u}_{\varepsilon}=0 . & \end{cases}
$$

We notice that the first term is the fidelity term with respect to the measured boundary datum, the other three terms are the regularization. Here the regularization is based on the so-called Mumford-Shah functional [9], and its approximation, in the sense of $\Gamma$-convergence, by phasefield functionals due to Ambrosio and Tortorelli $[2,3]$. In fact, the last three terms correspond to the Ambrosio-Tortorelli functional. We recall that in the Ambrosio-Tortorelli approach the discontinuous function $u$ and its discontinuity set $J(u)$ are replaced, respectively, by a smooth function $u$ and a smooth phase-field function $v$. Therefore, the Ambrosio-Tortorelli functional depends on two independent variables, the state variable $u$ and the phase-field variable $v$. To have an easier implementation and faster computations, we use here a formulation depending on a single variable only, namely the phase-field variable $v$, with the state variable $u$ depending on the phasefield variable $v$ through (1.5).

The link with the prescribed boundary datum and with the presence of cracks is through $\tilde{u}_{\varepsilon}$, the solution of the weighted elliptic equation. We observe that the single-well potential $V$ forces the phase-field function $v=1-\tilde{v}$ to be equal to 1 except in a small region, which is where the crack should be located. The tuning parameters $a, b$ and $c$ allow one to put more emphasis on one or the other of the features of the functional. Namely, $a$ controls the match with the Dirichlet datum, $b$ the smoothness of the reconstructed potential away from its discontinuities, and $c$ the penalization on the $(N-1)$-dimensional measure of the discontinuities. Therefore $c$ may be seen as a regularization parameter.

For the material loss case, we simply replace the single-well potential $V$ with the double-well potential $W$. Namely, we define $\mathcal{G}_{\varepsilon}: H(\Omega) \rightarrow \mathbb{R}$ in an analogous way by simply replacing $V$ with $W$, that is, for any $\tilde{v} \in H(\Omega)$, we set

$$
\mathcal{G}_{\varepsilon}(\tilde{v})=\frac{a}{\varepsilon^{q_{1}}} \int_{\gamma}\left|\tilde{u}_{\varepsilon}-g_{\varepsilon}\right|^{2}+b \int_{\Omega} \psi_{\varepsilon}(v)\left|\nabla \tilde{u}_{\varepsilon}\right|^{2}+\frac{c^{2}}{\varepsilon} \int_{\Omega} W(v)+\varepsilon \int_{\Omega}|\nabla v|^{2} .
$$

We then minimize the functional $\mathcal{G}_{\varepsilon}$ with respect to the phase-field variable $\tilde{v} \in H(\Omega)$ with the constraint $0 \leqslant \tilde{v} \leqslant 1$.

Here we use a more classical perimeter penalization and its approximation, again in the sense of $\Gamma$-convergence, with phase-field functionals due to Modica and Mortola [8]. In fact the last two 
terms are the Modica-Mortola functional, which corresponds to a penalization of the perimeter of $G_{K_{0}}$ in $\Omega$. Here the double-well potential $W$ forces the phase-field function $v$ to be either 0 (inside the material loss) or 1 (outside the material loss), with a quick transition between these two regions. We notice that the single-well potential $V$ forces the phase-field function $v$ to be close to 1 except in a small neighborhood of an $(N-1)$-dimensional region, that is, a crack or the boundary of a cavity, whereas the double-well potential allows the phase-field function $v$ to be close to 1 and to 0 on large regions, corresponding respectively to the exterior and the interior of a cavity. Therefore, the first method is apt for the recovery of crack-like structures while the second may be used for the recovery of material losses.

Summarizing, we shall minimize the functional $\mathcal{F}_{\varepsilon}$ when we aim to reconstruct defects such as cracks, and the functional $\mathcal{G}_{\varepsilon}$ when we aim to reconstruct material losses. Namely, we wish to solve numerically the following minimization problems (depending on the properties of the unknown defect $\left.K_{0}\right)$ :

(i) $\min \mathcal{F}_{\varepsilon}$ on $H(\Omega)$ with the constraint $0 \leqslant \tilde{v} \leqslant 1$ if $K_{0}$ contains portions of cracks,

(ii) $\min \mathcal{G}_{\varepsilon}$ on $H(\Omega)$ with the constraint $0 \leqslant \tilde{v} \leqslant 1$ if $K_{0}$ is a material loss defect.

Let us notice that, by the direct method, both these minimum problems admit a solution.

The motivation for using this approach is contained in the convergence analysis developed in $[10,11]$. For what concerns the cavity case, in Theorem 4.2 of [11] it has been proved, essentially by $\Gamma$-convergence techniques, that as the noise level $\varepsilon$ goes to 0 , a minimizer $\tilde{v}_{\varepsilon}$ to $\mathcal{G}_{\varepsilon}$ converges to the characteristic function of $K_{0}$, provided $K_{0}$ is a material loss.

For the crack case, in [10] a regularization based on the phase-field functionals due to Ambrosio and Tortorelli has been introduced. In that formulation, which is amenable to numerical implementation, the functionals to be minimized depend on two independent variables, the state variable $u$ and the phase-field variable $v$. In [10, Theorem 4.6] an analogous convergence result has been proved for this approach to the inverse crack problem. To have an easier implementation and faster computations, it would be desirable to have a formulation depending on a single variable only, namely on the phase-field variable $v$, and preserving the same convergence properties. This may be done in a rigorous way only for material losses (see [11]). The idea would be to let the state variable $u$ depend on the phase-field variable $v$, for instance through (1.5). This is the approach we have decided to follow in this paper. In fact our functional $\mathcal{F}_{\varepsilon}$ is a small modification of the one used in [10] with the additional constraint that $u$ is linked to $v$ through (1.5). Unfortunately, there is evidence that for the functional $\mathcal{F}_{\varepsilon}$ no convergence result holds (see the discussion in Section 5 of [11]). In the same section it is also discussed why, when letting $u$ depend on $v$, among other possible modifications of the functional developed in [10] the one used in this paper should be preferred. Finally, the numerical examples in this paper show that this simplification allows us to obtain good reconstruction with an easier implementation.

As the numerical method, in order to find the minimizers, we use an iterative method. We formulate the corresponding optimality system and we use a gradient method. In particular, in Section 2, we describe the method and we prove that the functional is decreasing along the iterations, unless we hit a critical point. Since the functional to be minimized is not convex, it is not possible to guarantee that we converge to an absolute minimizer, since there might be local minimizers or even critical points. In numerical experiments, we have used an Armijo-type line search to try to move past local minimizers or critical points that might be encountered along the iterations. An important feature of our approach and of our numerical simulations is that we do not need any a priori assumption about the number and the location of the defects (in the crack case, in principle, 
not even whether the defect is a crack or a cavity). Therefore, we do not make any initial guess on the number and location of the defects to be reconstructed. In fact in all the experiments we start with the same initial phase-field function $v=1-\tilde{v}$, which is a very small perturbation of the constant function equal to 1 . We cannot start with the function which is identically equal to 1 just because this is a critical point of the functional. Another important property of the method is that it works with few measurements. We recall that, at least in the planar case, two different measurements in principle suffice. Moreover, in the applications it is important to be able to obtain good reconstruction with as few measurements as possible. Therefore, we purportedly decided to use few measurements in our numerical simulations.

In Section 3 numerical simulations are presented for both the single-well and double-well approximations. Numerical experiments are performed for various types of defect with noise-free and noisy data-sets.

\section{Optimality system and the gradient method}

We now turn towards the numerical implementation of the method. In this section we describe the iterative algorithm we shall use in numerical experiments. In particular, we shall prove that the functional decreases along the iterations, unless we hit a critical point.

We begin by recalling the differentiability properties of the functionals $\mathcal{F}_{\varepsilon}$ and $\mathcal{G}_{\varepsilon}$, which have been investigated in [11, Section 6].

We define the following spaces. For any $p, 2 \leqslant p \leqslant+\infty$, let us set $L_{p}(\Omega)=\left\{\tilde{v} \in L^{p}(\Omega)\right.$ : $\tilde{v}=0$ a.e. in $\left.\Omega_{1}\right\}$ and $H_{p}(\Omega)=H^{1,2}(\Omega) \cap L_{p}(\Omega)$, with the norm $\|\tilde{v}\|_{L_{p}(\Omega)}=\|\tilde{v}\|_{L^{p}(\Omega)}$ and $\|\tilde{v}\|_{H_{p}(\Omega)}=\|\tilde{v}\|_{L^{p}(\Omega)}+\|\nabla \tilde{v}\|_{L^{2}(\Omega)}$. To any $\tilde{v} \in L^{2}(\Omega)$ we as usual associate the function $v=1-\tilde{v}$. If $\tilde{v}$ belongs either to $L_{p}(\Omega)$ or to $H_{p}(\Omega)$, then $v \in L^{p}(\Omega), v=1$ almost everywhere in $\Omega_{1}$, and provided $0 \leqslant \tilde{v} \leqslant 1$ almost everywhere in $\Omega$, we also have $0 \leqslant v \leqslant 1$ almost everywhere in $\Omega$. We observe that $H_{2}(\Omega)=H(\Omega)$ as previously defined.

For any $q \geqslant 2$, we define

$$
H_{\gamma}^{1, q}(\Omega)=\left\{u \in H^{1, q}(\Omega): \int_{\gamma} u=0\right\} .
$$

We observe that, by a generalized Poincaré inequality, on $H_{\gamma}^{1, q}(\Omega)$ the usual $H^{1, q}(\Omega)$ norm and the norm $\|u\|_{H_{\gamma}^{1, q}(\Omega)}=\|\nabla u\|_{L^{q}(\Omega)}$ are equivalent. Therefore, we shall set this second one as the natural norm on $H_{\gamma}^{1, q}(\Omega)$.

We define $\mathcal{H}_{\varepsilon}: L_{2}(\Omega) \rightarrow H_{\gamma}^{1,2}(\Omega)$ as follows:

$$
\mathcal{H}_{\varepsilon}(\tilde{v})=\tilde{u}_{\varepsilon}(\tilde{v}) \quad \text { for any } \tilde{v} \in L_{2}(\Omega) .
$$

There exist constants $p(\varepsilon) \geqslant 2$ and $q(\varepsilon)>2$, depending on $\varepsilon$ and $\alpha$, such that the following results hold.

First, $\mathcal{H}_{\varepsilon}: L_{2}(\Omega) \rightarrow H_{\gamma}^{1, q(\varepsilon)}(\Omega)$ with bounded image in $H_{\gamma}^{1, q(\varepsilon)}(\Omega)$, and, for any $\tilde{v}_{0} \in L_{2}(\Omega)$, $\mathcal{H}_{\varepsilon}$ is differentiable at $\tilde{v}_{0}$ with respect to the $L^{p}(\Omega)$ norm for all $p \geqslant p(\varepsilon)$, and the $H_{\gamma}^{1, q(\varepsilon)}(\Omega)$ norm. Let $D \mathcal{H}_{\varepsilon}\left(\tilde{v}_{0}\right): L_{p}(\Omega) \rightarrow H_{\gamma}^{1, q(\varepsilon)}(\Omega)$ be the differential at $\tilde{v}_{0}$. Then for any $\tilde{v}$ in $L_{p}(\Omega)$ we have

$$
D \mathcal{H}_{\varepsilon}\left(\tilde{v}_{0}\right)[\tilde{v}]=U_{\varepsilon}\left(\tilde{v}_{0}, \tilde{v}\right)
$$


where $U_{\varepsilon}=U_{\varepsilon}\left(\tilde{v}_{0}, \tilde{v}\right) \in H_{\gamma}^{1,2}(\Omega)$ solves the following problem:

$$
\begin{cases}\operatorname{div}\left(\psi_{\varepsilon}\left(v_{0}\right) \nabla U_{\varepsilon}\right)=\operatorname{div}\left(\psi_{\varepsilon}^{\prime}\left(v_{0}\right) \tilde{v} \nabla\left(\mathcal{H}_{\varepsilon}\left(\tilde{v}_{0}\right)\right)\right) & \text { in } \Omega \\ \psi_{\varepsilon}\left(v_{0}\right) \nabla U_{\varepsilon} \cdot v=0 & \text { on } \partial \Omega\end{cases}
$$

Obviously, $v_{0}=1-\tilde{v}_{0}$. We recall that for any vector-valued function $G \in L^{2}\left(\Omega, \mathbb{R}^{N}\right), \operatorname{div}(G)$ defines a functional on $H^{1,2}(\Omega)$ in the following way:

$$
\operatorname{div}(G)[\phi]=-\int_{\Omega} G \cdot \nabla \phi \quad \text { for any } \phi \in H^{1,2}(\Omega)
$$

Therefore, the weak formulation of (2.1) is to look for a function $U_{\varepsilon} \in H_{\gamma}^{1,2}(\Omega)$ such that

$$
\int_{\Omega} \psi_{\varepsilon}\left(v_{0}\right) \nabla U_{\varepsilon} \cdot \nabla \varphi=\int_{\Omega} \psi_{\varepsilon}^{\prime}\left(v_{0}\right) \tilde{v} \nabla\left(\mathcal{H}_{\varepsilon}\left(\tilde{v}_{0}\right)\right) \cdot \nabla \varphi \quad \text { for any } \varphi \in H^{1,2}(\Omega) .
$$

Here, and analogously throughout, the differentiability has to be understood in the following sense. For any $\tilde{v}$ in $L_{p}(\Omega)$,

$$
\mathcal{H}_{\varepsilon}\left(\tilde{v}_{0}+\tilde{v}\right)=\mathcal{H}_{\varepsilon}\left(\tilde{v}_{0}\right)+D \mathcal{H}_{\varepsilon}\left(\tilde{v}_{0}\right)[\tilde{v}]+R(\tilde{v})
$$

where

$$
\lim _{\|\tilde{v}\|_{L^{p}(\Omega)} \rightarrow 0} \frac{\|R(\tilde{v})\|_{H_{\gamma}^{1, q(\varepsilon)}(\Omega)}}{\|\tilde{v}\|_{L^{p}(\Omega)}}=0 .
$$

We conclude that, for any $\tilde{v}_{0} \in H(\Omega), \mathcal{F}_{\varepsilon}$ and $\mathcal{G}_{\varepsilon}$ are differentiable at $\tilde{v}_{0}$ with respect to the $H_{p}(\Omega)$ norm, for all $p \geqslant p(\varepsilon)$. Let $D \mathcal{F}_{\varepsilon}\left(\tilde{v}_{0}\right), D \mathcal{G}_{\varepsilon}\left(\tilde{v}_{0}\right): H_{p}(\Omega) \rightarrow \mathbb{R}$ be the differentials at $\tilde{v}_{0}$ of $\mathcal{F}_{\varepsilon}$ and $\mathcal{G}_{\varepsilon}$, respectively. Then, for any $\tilde{v} \in H_{p}(\Omega)$,

$$
\begin{aligned}
D \mathcal{F}_{\varepsilon}\left(\tilde{v}_{0}\right)[\tilde{v}]= & \frac{2 a}{\varepsilon^{q_{1}}} \int_{\gamma}\left(\mathcal{H}_{\varepsilon}\left(\tilde{v}_{0}\right)-g_{\varepsilon}\right) U_{\varepsilon}\left(\tilde{v}_{0}, \tilde{v}\right) \\
& +b \int_{\Omega}\left(2 \psi_{\varepsilon}\left(v_{0}\right) \nabla \mathcal{H}_{\varepsilon}\left(\tilde{v}_{0}\right) \cdot \nabla U_{\varepsilon}\left(\tilde{v}_{0}, \tilde{v}\right)-\psi_{\varepsilon}^{\prime}\left(v_{0}\right)\left|\nabla \mathcal{H}_{\varepsilon}\left(\tilde{v}_{0}\right)\right|^{2} \tilde{v}\right) \\
& +\frac{c^{2}}{\varepsilon} \int_{\Omega}\left(-V^{\prime}\left(v_{0}\right) \tilde{v}\right)+2 \varepsilon \int_{\Omega} \nabla \tilde{v}_{0} \cdot \nabla \tilde{v}
\end{aligned}
$$

and

$$
\begin{aligned}
D \mathcal{G}_{\varepsilon}\left(\tilde{v}_{0}\right)[\tilde{v}]= & \frac{2 a}{\varepsilon^{q_{1}}} \int_{\gamma}\left(\mathcal{H}_{\varepsilon}\left(\tilde{v}_{0}\right)-g_{\varepsilon}\right) U_{\varepsilon}\left(\tilde{v}_{0}, \tilde{v}\right) \\
& +b \int_{\Omega}\left(2 \psi_{\varepsilon}\left(v_{0}\right) \nabla \mathcal{H}_{\varepsilon}\left(\tilde{v}_{0}\right) \cdot \nabla U_{\varepsilon}\left(\tilde{v}_{0}, \tilde{v}\right)-\psi_{\varepsilon}^{\prime}\left(v_{0}\right)\left|\nabla \mathcal{H}_{\varepsilon}\left(\tilde{v}_{0}\right)\right|^{2} \tilde{v}\right) \\
& +\frac{c^{2}}{\varepsilon} \int_{\Omega}\left(-W^{\prime}\left(v_{0}\right) \tilde{v}\right)+2 \varepsilon \int_{\Omega} \nabla \tilde{v}_{0} \cdot \nabla \tilde{v}
\end{aligned}
$$

An important remark is the following. If $N=2$, then we may actually choose $p(\varepsilon)=2$, and we observe that $H_{2}(\Omega)$ is a Hilbert space, with the scalar product $\int_{\Omega} \nabla \tilde{v}_{1} \cdot \nabla \tilde{v}_{2}$ for any $\tilde{v}_{1}, \tilde{v}_{2} \in H_{2}(\Omega)$. 
If $N>2$, then it might happen that $p(\varepsilon)>2$ and that $H_{p(\varepsilon)}(\Omega)$ does not have a Hilbert space structure anymore. However, since $p(\varepsilon)$ is finite, $H_{p(\varepsilon)}(\Omega)$ is still a strictly convex real reflexive Banach space.

In what follows we shall fix $p=p(\varepsilon)$ (with $p(\varepsilon)=2$ if $N=2$ ), and we denote by $\mathcal{M F} \mathcal{F}_{\varepsilon}$ the functional defined on $H_{\gamma}^{1,2}(\Omega) \times H_{p(\varepsilon)}(\Omega)$ by

$$
\begin{array}{r}
\mathcal{M F}_{\varepsilon}(u, \tilde{v})=\frac{a}{\varepsilon^{q_{1}}} \int_{\gamma}\left|u-g_{\varepsilon}\right|^{2}+\int_{\Omega}\left(b \psi_{\varepsilon}(v)|\nabla u|^{2}+\frac{c^{2}}{\varepsilon} V(v)+\varepsilon|\nabla v|^{2}\right) \\
\text { for any }(u, \tilde{v}) \in H_{\gamma}^{1,2}(\Omega) \times H_{p(\varepsilon)}(\Omega) .
\end{array}
$$

This value is always finite. By a similar reasoning, for any $\left(u_{0}, \tilde{v}_{0}\right) \in H_{\gamma}^{1, q(\varepsilon)}(\Omega) \times H_{p(\varepsilon)}(\Omega)$, $\mathcal{M F}_{\varepsilon}$ is differentiable at $\left(u_{0}, \tilde{v}_{0}\right)$ and for any $(u, \tilde{v}) \in H_{\gamma}^{1,2}(\Omega) \times H_{p(\varepsilon)}(\Omega)$ we have

$$
\begin{aligned}
D \mathcal{M F}_{\varepsilon}\left(u_{0}, \tilde{v}_{0}\right)[(u, \tilde{v})]= & \frac{2 a}{\varepsilon^{q_{1}}} \int_{\gamma}\left(u_{0}-g_{\varepsilon}\right) u+b \int_{\Omega}\left(2 \psi_{\varepsilon}\left(v_{0}\right) \nabla u_{0} \cdot \nabla u-\psi_{\varepsilon}^{\prime}\left(v_{0}\right)\left|\nabla u_{0}\right|^{2} \tilde{v}\right) \\
& +\frac{c^{2}}{\varepsilon} \int_{\Omega}\left(-V^{\prime}\left(v_{0}\right) \tilde{v}\right)+2 \varepsilon \int_{\Omega} \nabla \tilde{v}_{0} \cdot \nabla \tilde{v}
\end{aligned}
$$

We observe that $\mathcal{F}_{\varepsilon}(\tilde{v})=\mathcal{M F}_{\varepsilon}\left(\mathcal{H}_{\varepsilon}(\tilde{v}), \tilde{v}\right)$. Analogously, we define $\mathcal{M G}_{\varepsilon}$ simply by replacing $V$ with $W$. Analogous properties of differentiability hold for $\mathcal{M} \mathcal{G}_{\varepsilon}$ as well.

Let us finally define $\mathcal{L F}_{\varepsilon}: H_{\gamma}^{1,2}(\Omega) \times H_{p(\varepsilon)}(\Omega) \times H^{1,2}(\Omega) \rightarrow \mathbb{R}$ by setting, for any $(u, \tilde{v}, \phi) \in$ $H_{\gamma}^{1,2}(\Omega) \times H_{p(\varepsilon)}(\Omega) \times H^{1,2}(\Omega)$,

$$
\mathcal{L F}_{\varepsilon}(u, \tilde{v}, \phi)=\mathcal{M F}_{\varepsilon}(u, \tilde{v})+\int_{\Omega} \psi_{\varepsilon}(v) \nabla u \cdot \nabla \phi-\int_{\partial \Omega} f_{\varepsilon} \phi .
$$

In an analogous way we define $\mathcal{L G}_{\varepsilon}$ by replacing $\mathcal{M F}_{\varepsilon}$ with $\mathcal{M G}_{\varepsilon}$.

We observe that $\mathcal{L F}_{\varepsilon}$ (and $\mathcal{L G}_{\varepsilon}$ as well) is differentiable at any $\left(u_{0}, \tilde{v}_{0}, \phi_{0}\right) \in H_{\gamma}^{1, q(\varepsilon)}(\Omega) \times$ $H_{p(\varepsilon)}(\Omega) \times H^{1,2}(\Omega)$. For any $(u, \tilde{v}, \phi) \in H_{\gamma}^{1,2}(\Omega) \times H_{p(\varepsilon)}(\Omega) \times H^{1,2}(\Omega)$ we have

$\frac{\partial \mathcal{L} \mathcal{F}_{\varepsilon}}{\partial u}\left(u_{0}, \tilde{v}_{0}, \phi_{0}\right)[u]=\frac{2 a}{\varepsilon^{q_{1}}} \int_{\gamma}\left(u_{0}-g_{\varepsilon}\right) u+2 b \int_{\Omega} \psi_{\varepsilon}\left(v_{0}\right) \nabla u_{0} \cdot \nabla u+\int_{\Omega} \psi_{\varepsilon}\left(v_{0}\right) \nabla \phi_{0} \cdot \nabla u$,

and

$$
\begin{aligned}
\frac{\partial \mathcal{L} \mathcal{F}_{\varepsilon}}{\partial \tilde{v}}\left(u_{0}, \tilde{v}_{0}, \phi_{0}\right)[\tilde{v}]= & -b \int_{\Omega} \psi_{\varepsilon}^{\prime}\left(v_{0}\right)\left|\nabla u_{0}\right|^{2} \tilde{v}+\frac{c^{2}}{\varepsilon} \int_{\Omega}\left(-V^{\prime}\left(v_{0}\right) \tilde{v}\right) \\
& +2 \varepsilon \int_{\Omega} \nabla \tilde{v}_{0} \cdot \nabla \tilde{v}-\int_{\Omega} \psi_{\varepsilon}^{\prime}\left(v_{0}\right) \tilde{v} \nabla u_{0} \cdot \nabla \phi_{0}
\end{aligned}
$$

and, finally,

$$
\frac{\partial \mathcal{L} \mathcal{F}_{\varepsilon}}{\partial \phi}\left(u_{0}, \tilde{v}_{0}, \phi_{0}\right)[\phi]=\int_{\Omega} \psi_{\varepsilon}\left(v_{0}\right) \nabla u_{0} \cdot \nabla \phi-\int_{\partial \Omega} f_{\varepsilon} \phi
$$

Then the resulting optimality system is the following. We look for critical points, or better minimizers, of $\mathcal{F}_{\varepsilon}$, or equivalently, of $\mathcal{M F}_{\varepsilon}(u, \tilde{v})$ subject to the constraint $u=\mathcal{H}_{\varepsilon}(\tilde{v})$. We use a 
gradient method; the algorithm is divided into several steps. A completely analogous method may be used for finding minimizers of $\mathcal{G}_{\varepsilon}$. We describe the algorithm and show that the functional is decreasing along the iterations.

Step 0: initialization. We initialize the algorithm by putting $k=0$ and choosing an initial guess $\tilde{v}_{0} \in H(\Omega)$ such that $0 \leqslant \tilde{v}_{0} \leqslant 1$ almost everywhere. We observe that taking $\tilde{v}_{0} \equiv 0$ (that is, $v_{0} \equiv 1$ ) is not a good choice because this is a critical point of the functional $\mathcal{F}_{\varepsilon}$, thus the gradient method fails in this case.

Step 1: finding $u_{k}$. We solve

$$
\begin{cases}\operatorname{div}\left(\psi_{\varepsilon}\left(v_{k}\right) \nabla u_{k}\right)=0 & \text { in } \Omega \\ \psi_{\varepsilon}\left(v_{k}\right) \nabla u_{k} \cdot v=f_{\varepsilon} & \text { on } \partial \Omega \\ \int_{\gamma} u_{k}=0 & \end{cases}
$$

that is, we look for $u_{k} \in H_{\gamma}^{1,2}(\Omega)$ such that

$$
\int_{\Omega} \psi_{\varepsilon}\left(v_{k}\right) \nabla u_{k} \cdot \nabla \phi-\int_{\partial \Omega} f_{\varepsilon} \phi=0 \quad \text { for any } \phi \in H^{1,2}(\Omega) .
$$

We notice that $u_{k}=\mathcal{H}_{\varepsilon}\left(\tilde{v}_{k}\right)$ and $u_{k}$ actually belongs to $H_{\gamma}^{1, q(\varepsilon)}(\Omega)$. By (2.6) and (2.9), for any $\tilde{\phi} \in H^{1,2}(\Omega)$ we have

$$
\mathcal{L F}_{\varepsilon}\left(u_{k}, \tilde{v}_{k}, \tilde{\phi}\right)=\mathcal{M} \mathcal{F}_{\varepsilon}\left(u_{k}, \tilde{v}_{k}\right)=\mathcal{F}_{\varepsilon}\left(\tilde{v}_{k}\right) \quad \text { and } \quad \frac{\partial \mathcal{L} \mathcal{F}_{\varepsilon}}{\partial \phi}\left(u_{k}, \tilde{v}_{k}, \tilde{\phi}\right)=0 .
$$

Step 2: finding $\phi_{k}$. We solve the following boundary value problem:

$$
\begin{cases}\operatorname{div}\left(\psi_{\varepsilon}\left(v_{k}\right) \nabla \phi_{k}\right)=-\operatorname{div}\left(2 b \psi_{\varepsilon}\left(v_{k}\right) \nabla u_{k}\right) & \text { in } \Omega, \\ \psi_{\varepsilon}\left(v_{k}\right) \nabla \phi_{k} \cdot v=-\frac{2 a}{\varepsilon^{q_{1}}}\left(u_{k}-g_{\varepsilon}\right) \chi_{\gamma} & \text { on } \partial \Omega, \\ \int_{\gamma} \phi_{k}=0 & \end{cases}
$$

Here $\chi_{\gamma}$ denotes the characteristic function of $\gamma$, that is,

$$
\left(u_{k}-g_{\varepsilon}\right) \chi_{\gamma}= \begin{cases}u_{k}-g_{\varepsilon} & \text { on } \gamma, \\ 0 & \text { on } \partial \Omega \backslash \gamma .\end{cases}
$$

The weak formulation of (2.12) is to look for $\phi_{k} \in H_{\gamma}^{1,2}(\Omega)$ such that

$$
\begin{aligned}
& \int_{\Omega} \psi_{\varepsilon}\left(v_{k}\right) \nabla \phi_{k} \cdot \nabla u=-2 b \int_{\Omega} \psi_{\varepsilon}\left(v_{k}\right) \nabla u_{k} \cdot \nabla u-\frac{2 a}{\varepsilon^{q_{1}}} \int_{\gamma}\left(u_{k}-g_{\varepsilon}\right) u \\
& \quad \text { for any } u \in H^{1,2}(\Omega) .
\end{aligned}
$$

Such a solution $\phi_{k}$ exists and is unique. Then $\mathcal{L F}_{\varepsilon}\left(u_{k}, \tilde{v}_{k}, \phi_{k}\right)=\mathcal{M} \mathcal{F}_{\varepsilon}\left(u_{k}, \tilde{v}_{k}\right)=\mathcal{F}_{\varepsilon}\left(\tilde{v}_{k}\right)$ and, by (2.7),

$$
\frac{\partial \mathcal{L} \mathcal{F}_{\varepsilon}}{\partial \phi}\left(u_{k}, \tilde{v}_{k}, \phi_{k}\right)=0 \quad \text { and } \quad \frac{\partial \mathcal{L} \mathcal{F}_{\varepsilon}}{\partial u}\left(u_{k}, \tilde{v}_{k}, \phi_{k}\right)=0
$$


Step 3: computing the gradient and updating $v_{k}$. We compute the differential of $\mathcal{F}_{\varepsilon}$ at the point $\tilde{v}_{k}$. We observe that if $u=\mathcal{H}_{\varepsilon}(\tilde{v})$, then for any $\tilde{\phi} \in H^{1,2}(\Omega)$ we have

$$
\mathcal{F}_{\varepsilon}(\tilde{v})=\mathcal{M F}_{\varepsilon}\left(\mathcal{H}_{\varepsilon}(\tilde{v}), \tilde{v}\right)=\mathcal{L F}_{\varepsilon}\left(\mathcal{H}_{\varepsilon}(\tilde{v}), \tilde{v}, \tilde{\phi}\right) .
$$

Therefore, since $u_{k}=\mathcal{H}_{\varepsilon}\left(\tilde{v}_{k}\right)$, and if we pick $\tilde{\phi}=\phi_{k}$, then

$$
D \mathcal{F}_{\varepsilon}\left(\tilde{v}_{k}\right)=\frac{\partial \mathcal{L} \mathcal{F}_{\varepsilon}}{\partial \tilde{v}}\left(u_{k}, \tilde{v}_{k}, \phi_{k}\right) .
$$

We conclude that, by (2.8), for any $\tilde{v} \in H_{p(\varepsilon)}(\Omega)$ we have

$$
\begin{aligned}
D \mathcal{F}_{\varepsilon}\left(\tilde{v}_{k}\right)[\tilde{v}]= & -b \int_{\Omega} \psi_{\varepsilon}^{\prime}\left(v_{k}\right)\left|\nabla u_{k}\right|^{2} \tilde{v}+\frac{c^{2}}{\varepsilon} \int_{\Omega}\left(-V^{\prime}\left(v_{k}\right) \tilde{v}\right) \\
& +2 \varepsilon \int_{\Omega} \nabla \tilde{v}_{k} \cdot \nabla \tilde{v}-\int_{\Omega} \psi_{\varepsilon}^{\prime}\left(v_{k}\right) \tilde{v} \nabla u_{k} \cdot \nabla \phi_{k} .
\end{aligned}
$$

Let us now consider the space $H_{p(\varepsilon)}(\Omega)$. We recall that either $H_{p(\varepsilon)}(\Omega)=H_{2}(\Omega)$ (if $N=2$ ), that is, $H_{p(\varepsilon)}(\Omega)$ is a Hilbert space, or $H_{p(\varepsilon)}(\Omega)$ is a strictly convex real reflexive Banach space (if $N>2$ ). In either case, if $H=H_{p(\varepsilon)}(\Omega)$, we fix an operator $T: H^{*} \rightarrow H$ such that for any $w^{*} \in H^{*}$, we have

$$
\left\langle w^{*}, T\left(w^{*}\right)\right\rangle=\left\|w^{*}\right\|^{2} \quad \text { and } \quad\left\|T\left(w^{*}\right)\right\|=\left\|w^{*}\right\|,
$$

where $\langle\cdot, \cdot\rangle$ is the usual duality between $H^{*}$ and $H$. We may choose $T$ as the duality mapping from $H^{*}$ into $H^{* *}=H$. If $H$ is a Hilbert space and we also identify $H^{*}$ with $H$, then $T$ is actually the identity. See, for instance, [12, Section 42.6]. Let $T_{\varepsilon}$ denote the corresponding operator for $H_{p(\varepsilon)}(\Omega)$.

For a positive constant $t_{k}$, we then update $\tilde{v}_{k}$ by setting

$$
\hat{v}_{k+1}=\tilde{v}_{k}-t_{k} T_{\varepsilon}\left(D \mathcal{F}_{\varepsilon}\left(\tilde{v}_{k}\right)\right) \text {. }
$$

We observe the following. If $D \mathcal{F}_{\varepsilon}\left(\tilde{v}_{k}\right)=0$, then $\left(u_{k}, \tilde{v}_{k}, \phi_{k}\right)$ is a critical point of $\mathcal{L} \mathcal{F}_{\varepsilon}$ and $\tilde{v}_{k}$ is a critical point of $\mathcal{F}_{\varepsilon}$, and the algorithm comes to a stop. Otherwise, provided $t_{k}$ is small enough, an easy computation shows that $\mathcal{F}_{\varepsilon}\left(\hat{v}_{k+1}\right)<\mathcal{F}_{\varepsilon}\left(\tilde{v}_{k}\right)$.

Step 4: normalization and finding $\tilde{v}_{k+1}$. We normalize $\hat{v}_{k+1}$ by truncation as follows. We set $\tilde{v}_{k+1}=\left(\hat{v}_{k+1} \wedge 1\right) \vee 0$. Then $\tilde{v}_{k+1} \in H_{p(\varepsilon)}(\Omega)$ and $0 \leqslant \tilde{v}_{k+1} \leqslant 1$ almost everywhere in $\Omega$.

Let us note that, by our hypotheses, such a truncation does not increase the value of the functional: in fact, for any $\hat{v} \in H_{p(\varepsilon)}(\Omega)$, if $\tilde{v}=(\hat{v} \wedge 1) \vee 0$, then

$$
\mathcal{F}_{\varepsilon}(\tilde{v}) \leqslant \mathcal{F}_{\varepsilon}(\hat{v})
$$

Therefore, we have found that either $D \mathcal{F}_{\varepsilon}\left(\tilde{v}_{k}\right)=0$, and the algorithm stops, or otherwise, provided $t_{k}$ is small enough, $\mathcal{F}_{\varepsilon}\left(\tilde{v}_{k+1}\right)<\mathcal{F}_{\varepsilon}\left(\tilde{v}_{k}\right)$.

Once we have computed $\tilde{v}_{k+1}$, we iterate the algorithm by going back to Step 1 .

\section{Numerical experiments}

The data for the numerical experiments are generated by solving the Laplace equation numerically on a domain with certain prescribed defects (cracks or cavities). We solve the Neumann problem with given flux on the boundary of the computational domain, and read off the corresponding 
Dirichlet data to get a feasible pair of Neumann and Dirichlet boundary data on a discrete set of measurement points on the boundary from which the defect has to be reconstructed. As input fluxes we choose pairs of plus-shaped current profiles with opposite sign located at two different sides of the rectangular computational domain. The Laplace equation is solved on a very fine irregular grid using linear finite elements. The boundary data are genuinely defined on the unevenly distributed nodal points of elements on the boundary and are interpolated onto a much courser regular grid of measurement points. When experimenting with noisy input data, both boundary values are contaminated by adding Gaussian distributed artificial noise to the data, usually with different noise levels for $f=\left.\frac{\partial u}{\partial \nu}\right|_{\gamma}$ and $g=\left.u\right|_{\gamma}$.

In Step 0 of our algorithm, we choose an initial guess $\tilde{v}_{0}$ which is a small perturbation of the function which is identically equal to 0 . We recall that $\tilde{v}_{0} \equiv 0$ has to be avoided because this is a critical point for our functionals. The initial guess is kept fixed in all the experiments and carries no information whatsoever on the defects to be reconstructed.

For the numerical implementation of Step 1 (that is, the numerical solution of equation (2.11) for $u_{k}$ with given $v_{k}$ and prescribed $f_{\varepsilon}$ ), we also use linear finite elements for the discretization of $u_{k}$. In contrast to the data generation routine, we discretize the potential on a regular, structured grid, which is usually much coarser than the grid used for the data generation. Later on, we shall assume that the phase-field $v_{k}$ is also an element of the space of piecewise linear functions on the same underlying regular grid as for $u_{k}$. To assemble the stiffness matrix for (2.11), however, we replace the phase-field $v_{k}$ by its $L^{2}$-projection onto the space of functions which are piecewise constant on the triangles of the finite element space. A completely analogous procedure is applied for the solution of the adjoint equation (2.13) described in Step 2 for the adjoint variable $\phi_{k}$. Note that both systems share the same stiffness matrix and the right-hand side of (2.13) can be easily assembled using a slightly modified stiffness matrix. We shall use up to six different Cauchy data-sets for the reconstruction of the defect. The data-sets correspond to all possible combinations of pairs of electrodes where each electrode is located on a different side of the computational rectangle. We can use the same factorization of the stiffness matrix for all different right-hand sides of (2.11) and (2.13).

The calculation of the descent direction for the cost functional as described in Step 3 requires another solution of an elliptic boundary value problem for the variable $\delta \tilde{v}_{k}=T_{\varepsilon}\left(D \mathcal{F}_{\varepsilon}\left(\tilde{v}_{k}\right)\right)$. As mentioned above, the update $\delta \tilde{v}_{k}$ is discretized using linear triangular elements on a regular grid. To find $\delta \tilde{v}_{k}$ we have to solve an elliptic equation with system matrix defined by a discretization of the operator $T: H^{*} \rightarrow H$. In our two-dimensional test examples, we always set $H=H^{1,2}(\Omega)$ and for any $w^{*} \in H^{*}$ we set $T\left(w^{*}\right)=v$ where $v$ solves $v-c \Delta v=w^{*}$ in a weak sense with some parameter $c>0$ and homogeneous Dirichlet boundary conditions. The choice of Dirichlet boundary conditions is motivated by the desire to keep the value of the phase-field constantly 1 on the boundary. The assembling of the right-hand side of the equation for $\delta \tilde{v}_{k}$ is done by evaluating (2.14) for functions $\tilde{v}$ piecewise linear in all bases.

The projection required in Step 4 is easily implemented for piecewise linear functions by thresholding the nodal values. Moreover, a suitable step-length for the update of the phase-field is found using an Armijo-type line search. We use a maximum number of five reduction steps for the correction of the step-length. Since each evaluation of the cost functional requires one solution of the state equation, we try to steer the step-size modification in a rather conservative way.

Within this setup, the following numerical experiments have been performed. For all experiments, the phase-field parameter $\varepsilon$ was decreased in several steps from an initial value of 
$\varepsilon=2 \cdot 10^{-4}$ down to $\varepsilon=1 \cdot 10^{-6}$ for the single-well potential and to $\varepsilon=2 \cdot 10^{-6}$ for the double-well case. We run 2500 iterations of our algorithm in the single-well case and 1000 in the double-well case. Figure 2 shows the final phase-field together with the linear crack (a white line) which was used for the data generation. We use all six available data-sets with electrode positions on (up/down), (left/right), (down/left), (up/left), (down/right), and (up/right) sides of the rectangle for the reconstruction and set the noise-level to zero. In this simple situation where the crack is located rather close to the boundary we obtain very good reconstruction of the crack location with the single-well approximation.

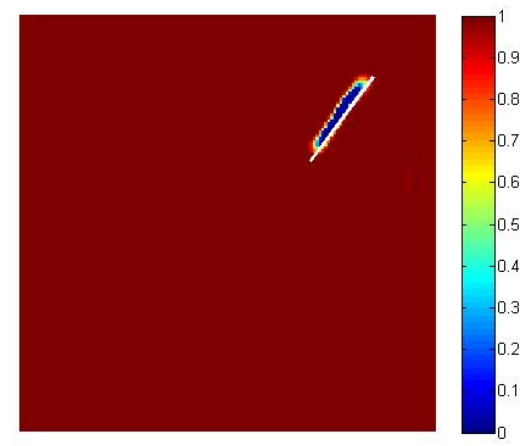

FIG. 2. Reconstruction of a small linear crack with noise-free data.

Figure 3 shows a comparison between reconstructions using three measurements (left image) with electrode positions on (left/right), (left/up), and (right/up) edges and six measurements (right figure), again in the single-well case. It is notable that in the reconstruction with three data-sets the crack tips are accurately identified but the reconstructed crack is strongly curved, which is probably due to the fact that we have no electrode located on the lower edge of the computational domain. In contrast the overall geometrical shape of the crack is reconstructed much better with six data-sets but the position of the crack tips is less accurate. We believe that this is due to the fact that, in general,
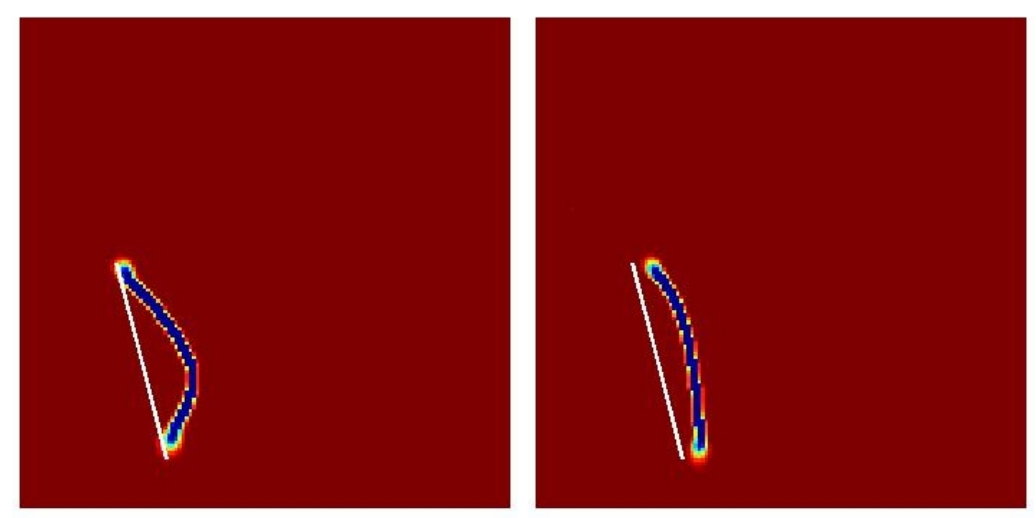

FIG. 3. Comparison of reconstructions from three and six measurements. 
the reconstructions tend to be offset towards the center of the computational domain, maybe because we keep the phase-field value fixed near the boundary. This tendency, together with the fact that the jump set of minimizers of the Mumford-Shah functional is smooth outside critical points, explains why when the shape recovered is more straight we have higher inaccuracy in the recovery of the crack tips. In any case, the main reason for these inaccuracies is the exponential ill-posedness of the problem, that is, even if the reconstructions are quite different from the looked-for defects, their corresponding boundary data may still match the measured ones very well. In these two simulations we added one percent of normally distributed noise to Neumann and Dirichlet data.

Figure 4 shows results for a situation with two cracks and different noise levels. Here we fixed the noise level for the Neumann data to be $1 \%$ for both experiments whereas the Dirichlet data were contaminated with $1 \%$ (left image) and $5 \%$ (right image) of noise. We used three measurements (left/right), (left/up), (right/up) and the single-well potential. There is no big difference in the quality of the reconstructions. In both cases the placement of the smaller crack in the upper right corner is inaccurate and the larger crack in the lower left corner is curved. Nonetheless the convergence of the algorithm is not heavily affected by the presence of (moderately strong) noise and the reconstructions are stable.
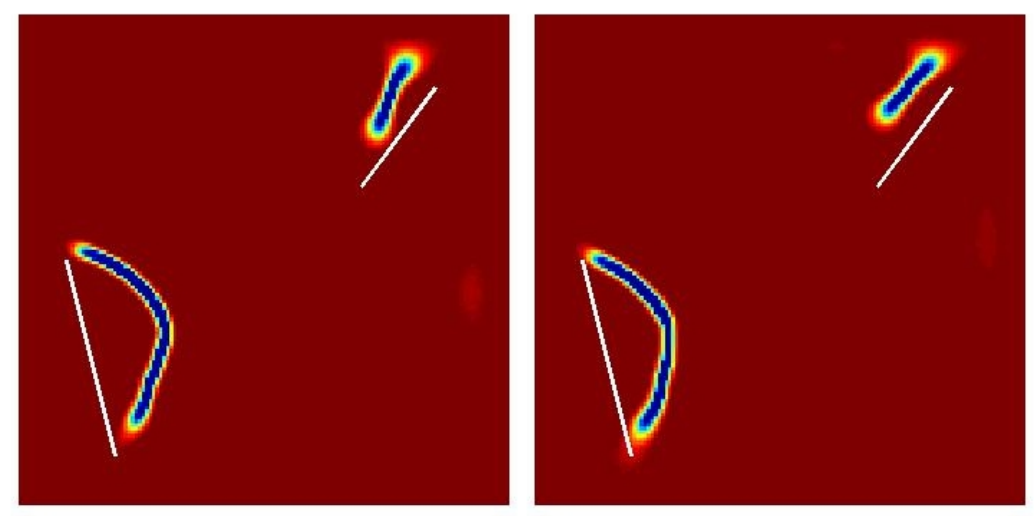

FIG. 4. Comparison with different noise levels.

We notice that the tendency of the reconstructed lower left crack to bend inward is not due to the choice of the mesh used. To see this, in the next Figure 5 we illustrate the dependence of the method on the mesh. In the leftmost image we have reconstruction with a low resolution mesh $(100 \times 100$, which is slightly lower than $140 \times 140$ used in the other experiments). In the center image we have a higher resolution mesh of $200 \times 200$, whereas in the rightmost image we have the same high resolution but the grid is rotated through 90 degrees. In these three experiments we used exactly the same Cauchy data with a noise level of $1 \%$ on both Neumann and Dirichlet data. The differences between these reconstructions are again due to the ill-posedness of the problem, whereas there is no significant influence of mesh refinement or rotation.

The next series of experiments presented in Figure 6 shows the tendency of the single-well based algorithm to produce dendrite-like structures. In fact, the dendrite-shaped crack in the leftmost image is reconstructed quite well. The polygonal crack in the middle image is approximated by a cloth-hanger-like structure which has a satisfactory data fit with a shorter overall length than the 

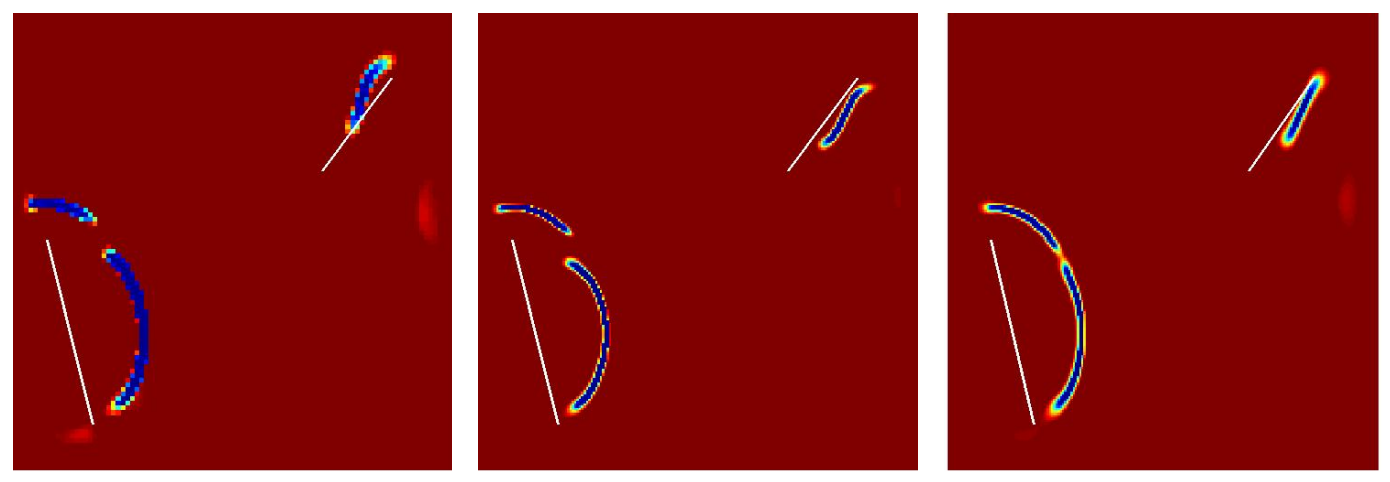

FIG. 5. Dependence of reconstructions from the mesh.

polygonal curve. Finally the cavity in the rightmost image is approximated by a one-dimensional structure which looks roughly like the skeleton of the cavity. In all these three experiments, the noise level is $1 \%$ for Neumann data and $5 \%$ for Dirichlet data and the three measurements (left/right), (left/up), (right/up) are used. The dendrite-like structures may be explained as follows. The method tries to minimize the length of the defects, thus it prefers those skeleton-like structures. Moreover, we already noticed that the jump set of minimizers of the Mumford-Shah functional is usually smooth outside critical points, thus sharp corners tend to be avoided. On the other hand, at a critical point of the jump set of minimizers of the Mumford-Shah functional, a typical structure which may be found is that of a triple point or propeller, which is exactly what we obtain in our reconstructions. Clearly, the most important reason of this behavior is again the exponential ill-posedness of the problem.
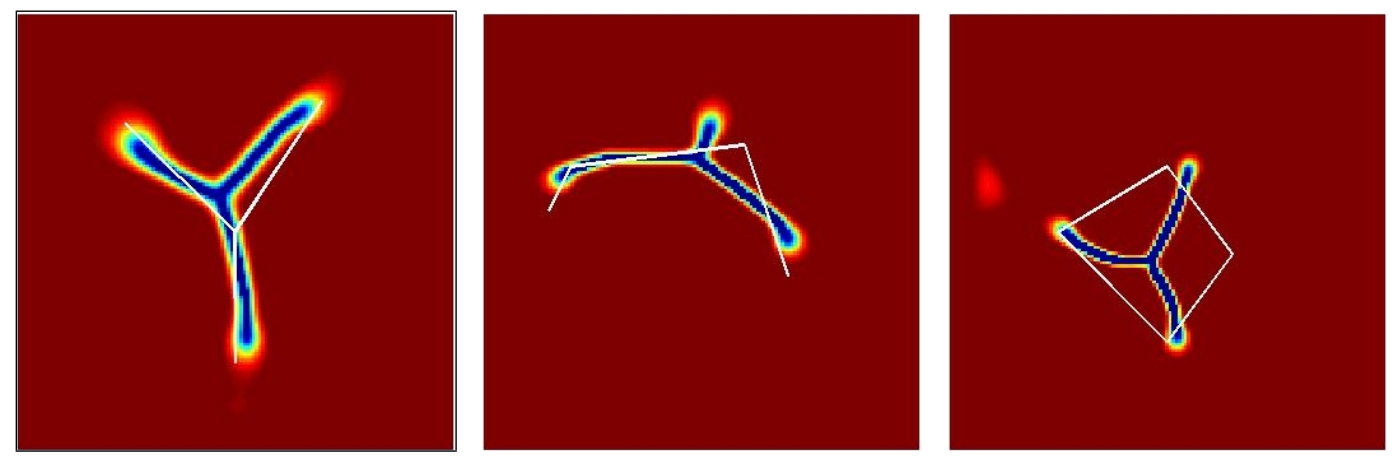

FIG. 6. Dendrite-like reconstructions with single-well potential.

Figure 7 shows reconstructions obtained by using the double-well approximation. As expected, the phase-field approximates the characteristic functions of one cavity (left image) and two cavities (right image). In these two tests, the noise level is $1 \%$ for Neumann data. In the left image, the noise level for Dirichlet data is 5\% and the three measurements (left/right), (left/up), (right/up) are used. In the right image, the noise level for Dirichlet data is $1 \%$ but only one measurement, namely (left/right), is used. The overall location of the cavities is satisfactory, but the lower left quadrilateral 

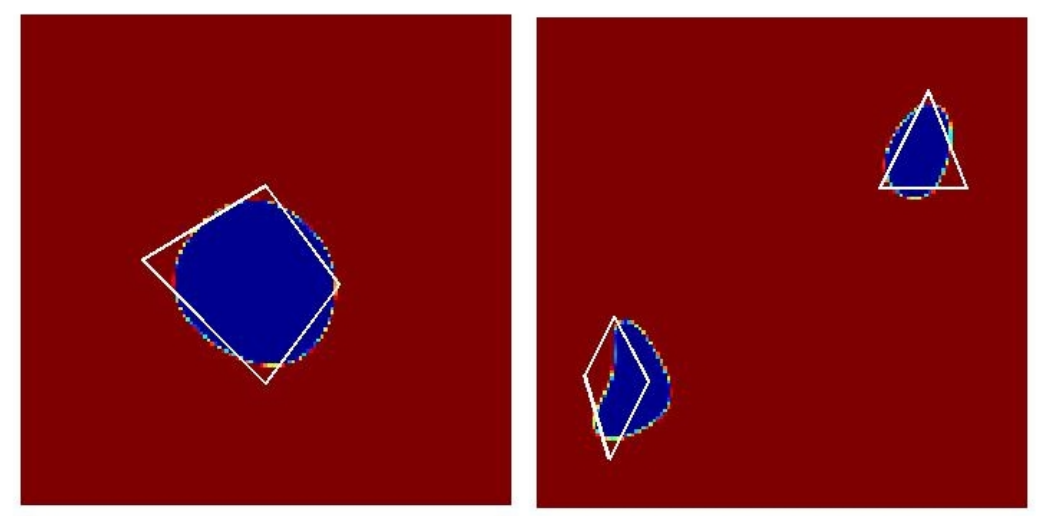

FIG. 7. Reconstructions of cavities with double-well potential.

is approximated by a non-convex shape. In this respect the experiment with the double-well potential for two cavities resembles the results shown in Figure 3 where the lower left crack also has a strong tendency to bend inward.

In another numerical experiment, documented in Figure 8, the double-well approach was used for the reconstruction of one-dimensional defects like the polygonal crack shown in the left image and the star-shaped crack shown in the right figure. In both cases the defect is approximated by a two-dimensional structure. An interesting feature is the occurrence of a self-intersection of the boundary curve of the reconstructed defect in the case of the star-shaped crack. Also in these two tests, the noise level is $1 \%$ for Neumann data and $5 \%$ for Dirichlet data and the three measurements (left/right), (left/up), (right/up) are used.
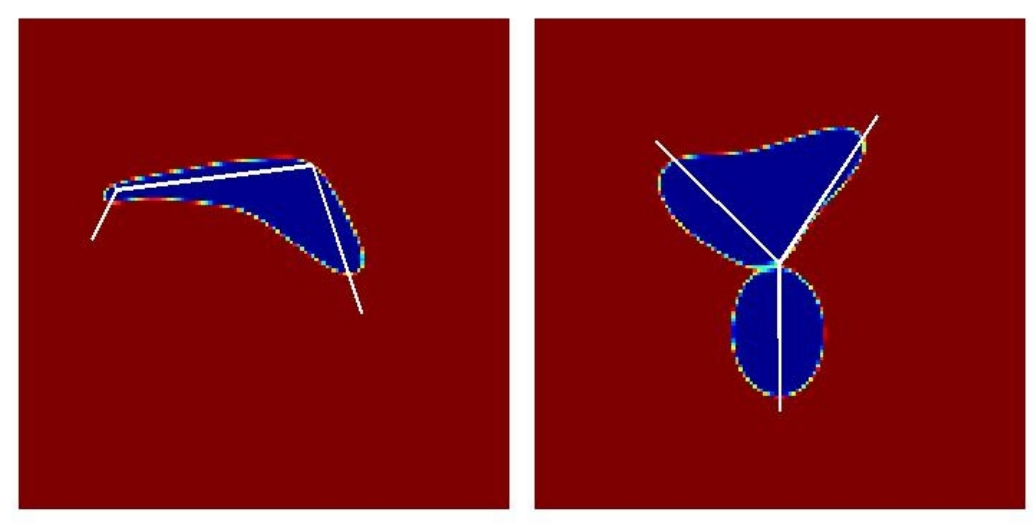

FIG. 8. Reconstructions of cracks with double-well potential.

Finally, we also tested the method when no defect is present. We used a noise level of $1 \%$ for both Neumann and Dirichlet data and a high resolution mesh. Figure 9 shows the final phase-field which is correctly identically equal to one but for some small artifacts in correspondence to the input pulses. We also compare the (numerical) solution $u$ to the direct problem (left image) to the 

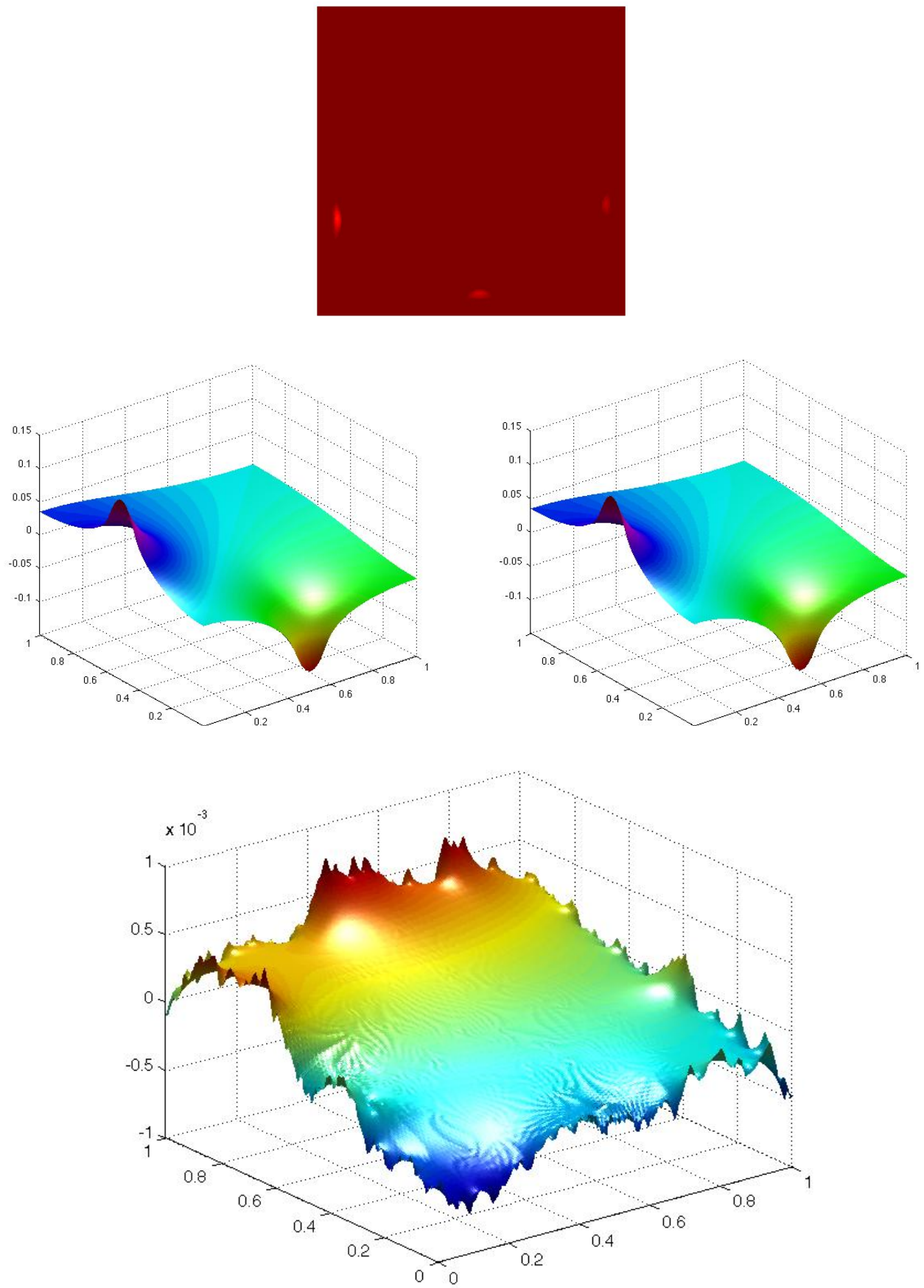

FIG. 9. No defect case. 
reconstructed $u$ by our iterative process (right image). Their difference, illustrated in the last image, shows that the deviation is comparable, in percentage, with the noise level on the Cauchy data used. This means that, although not designed for such a purpose, our approach provides indirectly a reasonably efficient method for the numerical solution of Cauchy problems.

As a conclusion we can state that both algorithms give reconstructions of the defects with a satisfactory accuracy for an exponentially ill-posed problem. The algorithms exhibit a quite stable behavior in the presence of data noise. The single-well and double-well models develop the types of structure for which they are designed (one-dimensional for the single-well and two-dimensional for the double-well potential), so the single-well approach approximates cavities by dendrites and the double-well approach approximates cracks by cavities. The double-well approach looks more stable with respect to noise, is slightly less sensitive to the adjustment of the phase-field parameter $\varepsilon$ and usually needs fewer iterations for convergence. This may be in accordance with the theory, in fact for the double-well case a convergence analysis is proved, whereas the single-well model we use is a modification of the one for which we have convergence results. Finally, it turned out to be important to update the phase-field parameter $\varepsilon$ adaptively during the algorithm. If the parameter $\varepsilon$ is chosen too small initially or decreased too fast, sharp interfaces develop too early, sometimes at incorrect locations, and the algorithm is not able to move well established interfaces to other locations. On the other hand, if the parameter $\varepsilon$ is decreased too much, the term containing the potential might prevail and not well established defects, usually the smaller ones, may disappear.

\section{Acknowledgments}

The second author is partially supported by GNAMPA under 2008 and 2009 projects and by the Italian Ministry of University and Research under PRIN 2008 project. Part of this work was done during a visit of the first author to the University of Trieste, supported by GNAMPA under 2008 project, and during a visit of the second author at the University of Graz, supported by the Special Research Center "Mathematical Optimization and Applications in Biomedical Sciences". The authors also wish to thank Alfio Borzì for useful discussions.

\section{REFERENCES}

1. Ambrosio, L., Fusco, N., \& Pallara, D. Functions of Bounded Variation and Free Discontinuity Problems. Clarendon Press, Oxford (2000). Zbl 0957.49001 MR 1857292

2. Ambrosio, L., \& Tortorelli, V. M. Approximation of functionals depending on jumps by elliptic functionals via $\Gamma$-convergence. Comm. Pure Appl. Math. 43 (1990), 999-1036. Zbl 0722.49020 MR 1075076

3. Ambrosio, L., \& Tortorelli, V. M. On the approximation of free discontinuity problems. Boll. Un. Mat. Ital. B (7) 6 (1992), 105-123. Zbl 0776.49029 MR 1164940

4. Ammari, H., Garnier, J., Kang, H., PARK, W.-K., \& SølnA, K. Imaging schemes for perfectly conducting cracks. SIAM J. Appl. Math. 71 (2011), 68-91. Zbl pre05894939 MR 2765649

5. Ammari, H., KANG, H., LeE, H., \& PARK, W.-K. Asymptotic imaging of perfectly conducting cracks. SIAM J. Sci. Comput. 32 (2010), 894-922. Zbl 1210.35279 MR 2609345

6. Braides, A. Approximation of Free-Discontinuity Problems. Springer, Berlin (1998). Z Zbl 0909.49001 MR 1651773

7. BRyAn, K., \& Vogelius, M. S. A review of selected works on crack identification. In: C. B. Croke et al. (eds.), Geometric Methods in Inverse Problems and PDE Control, Springer, New York (2004), 25-46. Zbl 1062.35166 MR 2169901 
8. Modica, L., \& Mortola, S. Un esempio di $\Gamma^{-}$-convergenza. Boll. Un. Mat. Ital. B (5) 14 (1977), 285-299. Zbl 0356.49008 MR 0445362

9. Mumford, D., \& Shah, J. Optimal approximations by piecewise smooth functions and associated variational problems. Comm. Pure Appl. Math. 42 (1989), 577-685. Zbl 0691.49036 MR 0997568

10. Rondi, L. Reconstruction in the inverse crack problem by variational methods. Eur. J. Appl. Math. 19 (2008), 635-660. Zbl 1148.74026 MR 2463224

11. Rondi, L. Reconstruction of material losses by perimeter penalization and phase-field methods. J. Differential Equations 251 (2011), 150-175. Z Zbl 1217.35215

12. ZeIDler, E. Nonlinear Functional Analysis and its Applications III: Variational Methods and Optimization. Springer, New York (1985). Zbl 0583.47051 MR 0768749 